\title{
Tensor-Structured Preconditioners and Approximate Inverse of Elliptic Operators in $\mathbb{R}^{d}$
}

\author{
Boris N. Khoromskij
}

Received: 19 August 2008 / Revised: 14 March 2009 / Accepted: 16 April 2009 /

Published online: 3 September 2009

(C) Springer Science+Business Media, LLC 2009

\begin{abstract}
In the present paper we analyze a class of tensor-structured preconditioners for the multidimensional second-order elliptic operators in $\mathbb{R}^{d}, d \geq 2$. For equations in a bounded domain, the construction is based on the rank- $R$ tensor-product approximation of the elliptic resolvent $\mathcal{B}_{R} \approx(\mathcal{L}-\lambda I)^{-1}$, where $\mathcal{L}$ is the sum of univariate elliptic operators. We prove the explicit estimate on the tensor rank $R$ that ensures the spectral equivalence. For equations in an unbounded domain, one can utilize the tensor-structured approximation of Green's kernel for the shifted Laplacian in $\mathbb{R}^{d}$, which is well developed in the case of nonoscillatory potentials. For the oscillating kernels $e^{-i \kappa\|x\|} /\|x\|, x \in \mathbb{R}^{d}, \kappa \in \mathbb{R}_{+}$, we give constructive proof of the rank- $O(\kappa)$ separable approximation. This leads to the tensor representation for the discretized $3 \mathrm{D}$ Helmholtz kernel on an $n \times n \times n$ grid that requires only $O\left(\kappa|\log \varepsilon|^{2} n\right)$ reals for storage. Such representations can be applied to both the $3 \mathrm{D}$ volume and boundary calculations with sublinear cost $O\left(n^{2}\right)$, even in the case $\kappa=O(n)$.

Numerical illustrations demonstrate the efficiency of low tensor-rank approximation for Green's kernels $e^{-\lambda\|x\|} /\|x\|, x \in \mathbb{R}^{3}$, in the case of Newton $(\lambda=0)$, Yukawa $\left(\lambda \in \mathbb{R}_{+}\right)$, and Helmholtz $\left(\lambda=i \kappa, \kappa \in \mathbb{R}_{+}\right)$potentials, as well as for the kernel functions $1 /\|x\|$ and $1 /\|x\|^{d-2}, x \in \mathbb{R}^{d}$, in higher dimensions $d>3$. We present numerical results on the iterative calculation of the minimal eigenvalue for the $d$-dimensional finite difference Laplacian by the power method with the rank truncation and based on the approximate inverse $\mathcal{B}_{R} \approx(-\Delta)^{-1}$, with $3 \leq d \leq 50$.
\end{abstract}

Keywords Preconditioning · High dimensions · Boundary value problems · Spectral problems · Tensor approximation · Green's kernels · Elliptic resolvent

Dedicated to Prof. I. Gavrilyuk on the occasion of his 60th birthday.

Communicated by Christoph Schwab.

B.N. Khoromskij (凶)

Max-Planck-Institute for Mathematics in the Sciences, Inselstr. 22-26, 04103 Leipzig, Germany

e-mail: bokh@mis.mpg.de 
Mathematics Subject Classification (2000) 65F50 - 65F30 - 46B28 - 47A80

\section{Introduction}

The construction of efficient preconditioned iterative methods plays an important role in the numerical analysis of high-dimensional equations arising in modern engineering, physics, and chemistry. For example, we mention the multidimensional integral-differential equations, elliptic/parabolic boundary value problems posed in $\mathbb{R}^{d}, d \geq 2$ (which include the Hartree-Fock/Kohn-Sham equations in density functional theory), the time-dependent Schrödinger equation in quantum molecular dynamics, the Lippmann-Schwinger integral formulation of the electronic Schrödinger and Hartree-Fock equations, the Poisson-Boltzmann equation in protein modeling, the deterministic Boltzmann equation, as well as multiparametric stochastic PDEs.

In multidimensional applications, traditional numerical methods of linear complexity in the volume fail due to the so-called "curse of dimensionality" (Bellman). This effect can be relaxed or completely avoided by a systematic application of low rank tensor-structured representations of the arising multivariate functions and related operators. Applications of tensor methods for representation of classical Green's kernels and elliptic resolvent [2-4, 10, 12, 18, 22], multidimensional convolution [6, 19, $20]$ and many other quantities arising in electronic structure calculations [16, 21, 23] have demonstrated surprising efficiency. In some applications the a priori fixed tensor subspace via sparse grids leads to efficient algorithms in higher dimensions $[11,30]$.

The present paper is motivated by the fact that application of tensor formats for iterative solving of the elliptic boundary value and spectral problems in higher dimensions is essentially based on using preconditioned (nonlinear) iterations with tensor truncation (cf. $[3,4,13,15,19,24])$. In this way the construction of spectrally close tensor-structured preconditioners for the class of elliptic integral-differential operators in $\mathbb{R}^{d}$ is one of the building blocks in development of efficient numerical methods in the case $d \geq 3$.

The idea of tensor-structured approximation of the elliptic inverse was first addressed in [3, 4, 10]. Particular constructions based on the sinc-approximation [29] applied to the operators $(-\Delta+\lambda I)^{-\alpha}, \lambda \in \mathbb{R}_{+}, \alpha>0$ on a hypercube were considered in $[9,10,19]$. In the present paper we prove the explicit estimate on the tensor rank of sinc-quadrature-based, preconditioners that ensures spectral equivalence. Efficient tensor approximation of nonoscillatory free-space Green's kernels for different discretization schemes have been described [12, 16, 18, 26]. To the best of our knowledge, the low rank tensor-structured approximation for oscillating kernels has not yet been addressed in the literature (cf. the asymptotically optimal method in the volume size based on the fast multipole type representations [5, 6]). In the present paper we construct and prove the rank- $O(\kappa)$ separable approximation for the oscillating kernel $e^{-i \kappa\|x\|} /\|x\|, x \in \mathbb{R}^{d}, \kappa \in \mathbb{R}_{+}$. Such representations allow the $3 \mathrm{D}$ volume and boundary calculations with sublinear cost $O(|\log \varepsilon| \kappa n)$, even in the case $\kappa=O(n)$. Notice that methods presented in $[5,6]$ lead to the linear complexity in the volume $O\left(\kappa^{3} \log \kappa+C(|\log \varepsilon|)^{3}\right)$. 
The rest of the paper is organized as follows. In Sect. 2 we formulate the classes of boundary value and spectral problems in bounded/unbounded domains and formulate the tensor-truncated preconditioned iterative schemes. We also collect some basic definitions on the separable approximation of multivariate functions (tensors) and related operators (matrices). Then we introduce the class of tensor-structured preconditioners for elliptic operators of the second order. We also discuss possible applications of the separable approximation for the free-space Green's kernels in the so-called FEM-BEM coupling methods and in the Green function formulations. In Sect. 3, we analyze the tensor-product preconditioner in $(0,1)^{d}$. Lemma 3.1 shows the linear scaling in $d$, while Lemma 3.2 proves the explicit estimate on the tensor rank that ensures spectral equivalence. Then we focus on tensor-product approximation of the integral operators in $\mathbb{R}^{d}$ with oscillating kernels. We give constructive proof of the rank- $O(\kappa)$ separable approximation for the oscillating kernel function $e^{-i \kappa\|x\|} /\|x\|, x \in \mathbb{R}^{d}, \kappa \in \mathbb{R}_{+}$. This leads to the tensor representation with approximation error $\varepsilon>0$, for the discretized 3D Helmholtz fundamental solution on an $n \times n \times n$ grid that requires only $O\left(|\log \varepsilon|^{2} n^{2}\right)$ reals for storage.

We complete Sect. 3 with a report on the results of numerical experiments illustrating the efficiency of the low tensor-rank approximation for the class of nonoscillating kernels for $d \geq 3$, and for the oscillating Helmholtz kernel in 3D. Another numerical example shows the linear scaling in $d$ of the "truncated" power iteration (cf. [13]) applied to the spectral problem for the Laplacian in $(0,1)^{d}, d \leq 50$.

\section{Problem Setting and Description of Preconditioners}

\subsection{Problem Setting}

We consider preconditioning methods for solving boundary value and eigenvalue problems

$$
\Lambda u=f \quad \text { and } \quad \Lambda u=\lambda u
$$

with the elliptic differential operator $\Lambda$ of the form

$$
\Lambda:=-\operatorname{div}(A \nabla u)+V u .
$$

Here the operator $\Lambda$ maps as $\Lambda: H_{0}^{1}(\Omega) \rightarrow H^{-1}(\Omega)$, where $\Omega \subset \mathbb{R}^{d}$ is some bounded or unbounded tensor-product domain. The operator coefficients $A=$ $\left\{a_{i j}(x)\right\}_{i, j=1}^{d} \in C^{\infty}\left(\Omega, \mathbb{R}^{d \times d}\right)$ and $V \in C(\Omega)$ in (2.1)-(2.2) are supposed to provide the low tensor-rank approximation of the corresponding solutions in $H_{0}^{1}(\Omega)$ (see $[13,24])$.

Efficient numerical solvers for high-dimensional boundary value and eigenvalue problems can be based on systematic use of the tensor approximation methods (cf. $[3,4,10,12,14])$. This concept relies on several prerequisites:

- Representation of arising operators and functions in rank-structured tensor formats and the corresponding error analysis (separable approximation); 
- Efficient implementation of multilinear matrix-vector operations with truncation to fixed tensor rank (tensor truncation);

- Construction of rank-structured spectrally close preconditioners or approximate inverse operators and their use in special iterative solvers which are well suited for truncated iterations.

The last topic will be the focus of the present paper.

\subsection{Tensor-Structured Representation of Functions and Operators}

In this section we recall the commonly used tensor-structured formats for representing the high-order tensors considered as the elements of tensor-product Hilbert space [28].

Representation of tensors: A $d$ th order tensor $V=\left[v_{i_{1}, \ldots, i_{d}}: i_{\ell} \in I_{\ell}\right] \in \mathbb{R}^{\mathcal{I}}(\mathcal{I}=$ $\left.I_{1} \times \cdots \times I_{d}\right)$ is an element of the tensor-product Hilbert space $\mathbb{V}_{\mathbf{n}}=\bigotimes_{\ell=1}^{d} \mathbb{V}_{\ell}$ of real-valued (complex-valued) functions of the discrete argument, with $\mathbb{V}_{\ell}=\mathbb{R}^{I_{\ell}}$, and equipped with the Euclidean inner product $\langle\cdot, \cdot\rangle: \mathbb{V}_{\mathbf{n}} \times \mathbb{V}_{\mathbf{n}} \rightarrow \mathbb{R}$, where $\mathbf{n}=$ $\left(n_{1}, \ldots, n_{d}\right)$. In general, we have $I_{\ell}=\left\{1, \ldots, n_{\ell}\right\}$; however, for ease of discussion we set $\# I_{\ell}=n_{\ell}=n(\ell=1, \ldots, d)$. The concept of rank-structured tensor-product formats enables us to eliminate the exponential increase in the number of entries in $V$, as $n^{d}$. In this way, one considers the outer product of vectors $v^{(\ell)}=\left\{v_{i_{\ell}}^{(\ell)}\right\}_{i_{\ell} \in I_{\ell}} \in \mathbb{V}_{\ell}$ $(\ell=1, \ldots, d)$ that forms the canonical rank-1 tensor

$$
V \equiv\left[v_{\mathbf{i}}\right]_{\mathbf{i} \in \mathcal{I}}=v^{(1)} \otimes \cdots \otimes v^{(d)} \in \mathbb{V}_{\mathbf{n}} \quad \text { with entries } v_{\mathbf{i}}=v_{i_{1}}^{(1)} \cdots v_{i_{d}}^{(d)},
$$

requiring only $d n$ elements to store it (now linear in the dimension). In the case $d=2$, the outer product of vectors represents a rank-1 matrix. by

Rank-R canonical representation (tensor class $\mathcal{C}_{R, \mathbf{n}}$ ) of a tensor $V \in \mathbb{V}_{\mathbf{n}}$ is defined

$$
V=\sum_{\nu=1}^{R} \beta_{\nu} v_{v}^{(1)} \otimes \cdots \otimes v_{v}^{(d)}, \quad \beta_{\nu} \in \mathbb{R}
$$

with normalized vectors $v_{v}^{(\ell)} \in \mathbb{V}_{\ell}(\ell=1, \ldots, d)$. The minimal parameter $R$ in $(2.3)$ is called the rank (or canonical rank) of a tensor.

Tucker model (tensor class $\mathcal{T}_{\mathbf{r}, \mathbf{n}}, \mathbf{r}=\left(r_{1}, \ldots, r_{d}\right)$ ): Rank-r orthogonal Tucker approximation is based on subspaces $\mathbb{T}_{\mathbf{n}}:=\bigotimes_{\ell=1}^{d} \mathbb{T}_{\ell} \subset \mathbb{V}_{\mathbf{n}}$ for certain $\mathbb{T}_{\ell}=$ $\operatorname{span}\left\{t_{v_{\ell}}^{(\ell)}\right\}_{v_{\ell}=1}^{r_{\ell}} \subset \mathbb{V}_{\ell}$ with $r_{\ell}:=\operatorname{dim} \mathbb{T}_{\ell}<n$. Then each tensor $V \in \mathbb{T}_{\mathbf{n}}$ can be represented by a sum of rank-1 elements

$$
V=\sum_{v_{1}=1}^{r_{1}} \cdots \sum_{v_{d}=1}^{r_{d}} \beta_{v_{1}, \ldots, v_{d}} t_{v_{1}}^{(1)} \otimes \cdots \otimes t_{v_{d}}^{(d)} \equiv \boldsymbol{\beta} \times_{1} T^{(1)} \times_{2} T^{(2)} \cdots \times_{d} T^{(d)},
$$

where $T^{(\ell)}=\left[t_{1}^{(\ell)} \cdots t_{r_{\ell}}^{(\ell)}\right], t_{v_{\ell}}^{(\ell)} \in \mathbb{T}_{\ell}(\ell=1, \ldots, d)$ is the orthogonal matrix and $\times_{\ell}$ denotes the mode- $\ell$ contracted product. Here $r=\max _{\ell}\left\{r_{\ell}\right\}$ is called the Tucker rank (Trank). In our applications we have $r \ll n$, say $r=O(\log n)$. The coefficients tensor $\boldsymbol{\beta}=\left[\beta_{v_{1}, \ldots, v_{d}}\right] \in \mathbb{R}^{r_{1} \times \cdots \times r_{d}}$ (core tensor), is an element of the dual (reciprocal) tensor space $\mathbb{B}_{\mathbf{r}}$. 
Remark 2.1 Note that $\mathcal{C}_{R}$ is a subset of $\mathcal{T}_{\mathbf{r}}$ with $\mathbf{r}=(R, \ldots, R)$ corresponding to the case of diagonal core tensors. Hence we have the following lower bound on the canonical rank in (2.3), in terms of the Tucker rank in (2.4), $r=\max _{\ell} r_{\ell} \leq R$.

Mixed (two-level) Tucker-canonical model: Subclass $\mathcal{T}_{\mathcal{C}_{R, \mathbf{r}}} \subset \mathcal{T}_{\mathbf{r}, \mathbf{n}}$ with $\boldsymbol{\beta} \in$ $\mathcal{C}_{R, \mathbf{r}} \subset \mathbb{B}_{\mathbf{r}}$ consists of tensors in the form

$$
V=\left(\sum_{\nu=1}^{R} \beta_{\nu} b_{\nu}^{(1)} \otimes \cdots \otimes u_{v}^{(d)}\right) \times_{1} T^{(1)} \times_{2} T^{(2)} \cdots \times_{d} T^{(d)},
$$

where $T^{(\ell)} \in \mathbb{R}^{n \times r_{\ell}}(\ell=1, \ldots, d)$ is the orthogonal matrix.

Storage constraints: The storage requirements for the Tucker (resp. canonical) decomposition is bounded by $r^{d}+d r n$ (resp. $R+d R n$ ), where usually $r \ll n$ and $r \ll R$. Representing the $\mathcal{T}_{\mathcal{C}_{R, \mathbf{r}}}$ format amounts to $d R r+R+d r n$ reals (linear scaling in $d, n, R, r)$.

Representation of tensor-structured matrices (operators): The index sets $I_{1}, \ldots, I_{d}$ and $J_{1}, \ldots, J_{d}$ give rise to the pair of tensor-product Hilbert spaces $V=\mathbb{R}^{I_{1}} \otimes \cdots \otimes$ $\mathbb{R}^{I_{d}}$ and $W=\mathbb{R}^{J_{1}} \otimes \cdots \otimes \mathbb{R}^{J_{d}}$. Given matrices $A^{(\ell)} \in \mathbb{R}^{I_{\ell} \times J_{\ell}}(\ell=1, \ldots, d)$, their Kronecker product $\mathcal{A}:=A^{(1)} \otimes \cdots \otimes A^{(d)}$ is defined as the mapping

$$
\mathcal{A}: V \rightarrow W, \quad V \ni v=v^{(1)} \otimes \cdots \otimes v^{(d)} \mapsto \mathcal{A} v=A^{(1)} v^{(1)} \otimes \cdots \otimes A^{(d)} v^{(d)} \in W .
$$

We introduce a class $\mathcal{M}_{R, \mathbf{n}}$ of the Kronecker rank $R(\operatorname{Krank}(\mathcal{A})=R)$ matrices in the form

$$
\mathcal{A}=\sum_{\nu=1}^{R} A_{v}^{(1)} \otimes \cdots \otimes A_{v}^{(d)}, \quad A_{v}^{(\ell)} \in \mathbb{R}^{I_{\ell} \times J_{\ell}}, \ell=1, \ldots, d,
$$

where $R$ is supposed to be small, say $R=O(\log n)$. This provides a tremendous reduction in storage from $\mathcal{O}\left(n^{2 d}\right)$ to $\mathcal{O}\left(d R n^{2}\right)$. The matrices $A_{v}^{(\ell)}$ can be "compressed" by techniques of hierarchical or wavelet matrix approximation or adapting Toeplitz/circulant type structures (cf. the convolution with translation invariant kernels) reducing the complexity and storage to $\mathcal{O}(d R n \log n)$.

Low tensor-rank nonlinear approximation: Since both $\mathcal{T}_{\mathbf{r}}$ and $\mathcal{C}_{R}$ are not linear spaces, we arrive at a nontrivial nonlinear approximation problem

$$
A_{0} \in \mathbb{V}_{\mathbf{n}}: \quad A=\operatorname{argmin}_{T \in \mathcal{S}}\left\|A_{0}-T\right\|_{\mathbb{V}_{\mathbf{n}}}
$$

with $\mathcal{S} \in\left\{\mathcal{T}_{\mathbf{r}}, \mathcal{C}_{R}, \mathcal{T}_{\mathcal{C}_{R, \mathbf{r}}}\right\}$ or $\mathcal{S} \subset \mathcal{T}_{\mathbf{r}}$ being a subclass of symmetric, antisymmetric, or positive tensors. The replacement of $A_{0}$ by an approximation $A \in \mathcal{S}$ is called the tensor truncation to $\mathcal{S}$ and denoted by $T_{\mathcal{S}} A_{0}$. In practice, the computation of the minimizer $A$ can be performed only approximately.

In the case $\mathcal{S}=\mathcal{T}_{\mathrm{r}}$, we have proven the solvability of the minimization problem (2.7) and the quadratic convergence in the "energy functional," and we have also analyzed the structure of its Frechét derivative (cf. [18]). Basic ALS iteration to compute the Tucker approximation in $\mathcal{T}_{\mathbf{r}}$ was presented in [7, 8]. With a good initial guess, we normally observe the stable geometric convergence of the ALS method for orthogonal Tucker approximation. On the other hand, the (nonorthogonal) canonical 
decomposition in $\mathcal{C}_{R}$ is well suited for fast multilinear algebra. The mixed tensor format $\mathcal{T}_{\mathcal{C}_{R, \mathbf{r}}}$ combined with the multigrid acceleration techniques enables us to utilize the favorable features of both Tucker and canonical models [23].

\subsection{Equations in Bounded Domain}

We are interested in preconditioned iterations for solving the FEM approximations of the variational boundary value problem:

$$
\begin{aligned}
& \text { find } u \in H_{0}^{1}(\Omega) \\
& \text { such that } \int_{\Omega} \sum_{i, j=1}^{d} a_{i j}(x) \partial_{i} u \partial_{j} v+V u v=\int_{\Omega} f v, \quad \forall v \in H_{0}^{1}(\Omega) \text {, }
\end{aligned}
$$

as well as of the related eigenvalue problem: Find $(\lambda, u) \in \mathbb{R} \times H_{0}^{1}(\Omega) \backslash\{0\}$ such that

$$
\int_{\Omega} \sum_{i, j=1}^{d} a_{i j}(x) \partial_{i} u \partial_{j} v+V u v=\lambda \int_{\Omega} u v, \quad \forall v \in H_{0}^{1}(\Omega) .
$$

For numerical approximation and preconditioning of "tensor-structured" discrete elliptic operators defined in the hypercube $\Omega=(0,1)^{d}$, we consider the Galerkin schemes via the tensor-product piecewise polynomial basis functions $\left\{\phi_{\mathbf{i}}\right\}$,

$$
\phi_{\mathbf{i}}(x)=\prod_{\ell=1}^{d} \phi_{i_{\ell}}\left(x_{\ell}\right), \quad \mathbf{i} \in \mathcal{I}=I^{d}:=\{1, \ldots, n\}^{d} .
$$

For ease of presentation, $\phi_{i_{\ell}}$ are supposed to be linear polynomials in variable $x_{\ell}$, i.e., we choose the Galerkin subspace of the Courant hat functions $\mathbb{V}_{n}=\left(V_{n}\right)^{d} \subset$ $\left(H_{0}^{1}(0,1)\right)^{d}$ associated with the uniform tensor-product grid. The Galerkin approximation to the initial BVP (2.8) now reads as follows:

$$
\mathcal{L} U \equiv(\mathcal{A}+\mathcal{V}) U=F, \quad U \in \mathbb{R}^{\mathcal{I}},
$$

while the spectral problem (2.9) is discretized as

$$
\mathcal{L} U \equiv(\mathcal{A}+\mathcal{V}) U=\lambda \mathcal{M} U, \quad U \in \mathbb{R}^{\mathcal{I}},
$$

where $U$ denotes the vector representation of $u \in \mathbb{V}_{n}$ in the basis set $\left\{\phi_{\mathbf{i}}\right\}$. This leads to the explicit definition of the stiffness/mass matrices,

$$
\begin{aligned}
& \langle\mathcal{A} U, V\rangle:=\langle A \nabla u, \nabla v\rangle_{\left(L^{2}(\Omega)\right)^{d}}, \quad\langle\mathcal{V} U, V\rangle:=\langle V u, v\rangle_{L^{2}(\Omega)}, \\
& \langle\mathcal{M} U, V\rangle:=\langle u, v\rangle_{L^{2}(\Omega)},
\end{aligned}
$$

and the vector in the right-hand side,

$$
\langle F, V\rangle:=\langle f, v\rangle_{L^{2}(\Omega)}, \quad \forall u, v \in \mathbb{V}_{n} .
$$


We follow the concept of approximate (truncated) iterations based on usage of the rank-structured formats to represent matrix-vector operations in the framework of preconditioned iterative solvers (cf. [13, 15]).

For the linear system (2.11) the truncated preconditioned iteration takes the form

$$
\widetilde{U}_{m+1}=U_{m}-\mathcal{B}^{-1}\left(\mathcal{L} U_{m}-F\right), \quad U_{m+1}:=T_{\mathcal{S}}\left(\widetilde{U}_{m+1}\right),
$$

where the truncation operator $T_{\mathcal{S}}$ is defined by the nonlinear approximation procedure that "projects" the respective vectors onto some manifold $\mathcal{S}$ of rank-structured tensors.

In turn, solving the spectral problem (2.12) in the rank-structured formats can be realized via the truncated preconditioned inverse iteration as follows:

$$
\begin{gathered}
\widetilde{U}_{m+1}=U_{m}-\mathcal{B}^{-1}\left(\mathcal{L} U_{m}-\mu_{m} U_{m}\right), \quad U_{m+1}:=T_{\mathcal{S}}\left(\widetilde{U}_{m+1}\right), \\
U_{m+1}:=U_{m+1} /\left\|U_{m+1}\right\|, \quad \mu_{m+1}:=\left(\mathcal{L} U_{m+1}, U_{m+1}\right) .
\end{gathered}
$$

In both cases, the preconditioner $\mathcal{B}^{-1}$ can be chosen as inverse of the shifted Laplacian. In the following we present the more general construction of $\mathcal{B}$.

In the framework of FEM Galerkin equations (2.11) and (2.12) we introduce the class of elliptic operators which allow the low tensor rank-approximate inverse. Let $\Sigma_{0}: H_{0}^{1}(\Omega) \rightarrow H^{-1}(\Omega)$ be an elliptic operator given by the (positive) diagonal coefficients matrix $D=\operatorname{diag}\left\{D_{1}, \ldots, D_{d}\right\} \in C\left(\Omega ; \mathbb{R}^{d \times d}\right)$ and by the "univariate" potentials $V_{\ell}\left(x_{\ell}\right)$,

$$
\Sigma_{0}:=-\sum_{\ell=1}^{d} \Sigma_{\ell}, \quad \text { with } \Sigma_{\ell}=-\frac{d}{d x_{\ell}} D_{\ell}\left(x_{\ell}\right) \frac{d}{d x_{\ell}}+V_{\ell}\left(x_{\ell}\right), \ell=1, \ldots, d .
$$

Suppose that the elliptic operator $\Sigma_{\ell}(\ell=1, \ldots, d)$ is positive definite, and let $\mathcal{A}_{\ell} \in$ $\mathbb{R}^{n \times n}$ be the Galerkin discretization of $\Sigma_{\ell}$. Introduce the class of rank- $R$ Kronecker product preconditioners defined by approximate inverse

$$
\mathcal{B}_{R} \approx \mathcal{L}_{0}^{-1} \quad \text { with } \mathcal{L}_{0}:=\mathcal{A}_{1} \otimes \cdots \otimes I+\cdots+I \otimes \cdots \otimes \mathcal{A}_{d}
$$

Here the coefficients $D_{\ell}, V_{\ell}$ can be chosen from the optimization of the condition number in

$$
C_{1}\langle\mathcal{L} U, U\rangle \leq\left\langle\mathcal{L}_{0} U, U\right\rangle \leq C_{2}\langle\mathcal{L} U, U\rangle, \quad \forall U \in \mathbb{V}_{n},
$$

so that the matrix $\mathcal{L}_{0}$ is supposed to be spectrally close/equivalent to the initial stiffness matrix $\mathcal{A}+\mathcal{V}$.

The construction of an approximate inverse in the form of rank- $R$ Kronecker tensor-product representation is based on the sinc-quadrature method (see Lemma 3.2 below). In Sect. 3.1 we prove the spectral equivalence estimates for the preconditioner $\mathcal{B}_{R}$ and show that the storage requirement for the respective representation (3.1) is linear in $d, \mathcal{O}\left(d R n^{q}\right)$, with $q=1,2$ (compare with the linear complexity in the volume $\left.N=n^{d}\right)$. 
Remark 2.2 We notice that the potential function $V(x), x \in \mathbb{R}^{d}$ might include nonseparable (singular) terms like $\sum_{m} 1 /\left\|x-x_{m}\right\|$. The low tensor-rank approximation of a class of Green's kernels will be addressed in Sect. 2.4. Moreover, the tensor representation of the Schrödinger type operators was discussed in [3].

\subsection{Equations in Unbounded Domain and BEM Applications}

\subsubsection{General Discussion}

In the case $\Omega=\mathbb{R}^{d}$, we set $A=I \in C\left(\Omega ; \mathbb{R}^{d \times d}\right)$ and consider the corresponding boundary value problem (BVP) and eigenvalue problem (EVP) in the forms:

$$
-\Delta u+V u=f \quad \text { and } \quad-\Delta u+V u=\lambda u,
$$

subject to the corresponding "radiation condition" as $\|x\| \rightarrow \infty$. There are several commonly used numerical methods for solving (2.14) based on the application of the fundamental solutions of related elliptic operators with constant coefficients:

1. Computation of a particular solution (potential) by the convolution of the given right-hand side (density) with the related fundamental solution.

2. Boundary element methods (BEM) in bounded/unbounded domains.

3. Green function (fixed point) formulation of (2.14).

The reference elliptic operator with constant coefficients will be chosen as the shifted Laplacian

$$
-\Delta \pm z^{2} I, \quad z \in[0, \infty) .
$$

Hence, in general, we are interested in the tensor-structured approximation of the respective elliptic resolvent (Green's function operator)

$$
R_{z}:=\left(-\Delta \pm z^{2} I\right)^{-1}, \quad z \in[0, \infty),
$$

with the kernel function defined by the spherically symmetric fundamental solution (Green function) $G_{z}(x), x \in \mathbb{R}^{d}$. The fundamental solution of the operators $-\Delta$ and $-\Delta+z^{2}, \operatorname{Re} z^{2}>0$, in $\mathbb{R}^{d}$ is given by

$$
\begin{aligned}
& G_{0}(x)=\frac{\Gamma\left(\frac{d}{2}-1\right)}{4 \pi^{d / 2}\|x\|^{d-2}} \text { and } \\
& G_{z}(x)=\frac{1}{2 \pi^{d / 2}}\left(\frac{z}{\|x\|^{d-2}}\right)^{d / 2-1} K_{d / 2-1}(z\|x\|),
\end{aligned}
$$

respectively, where $K_{v}$ is the modified Bessel function of the second kind [1, Sect. 9.6]. In turn, the fundamental solution of the Helmholtz-type operator $\Delta+\kappa^{2}$, $\kappa^{2}>0$, takes the form

$$
G_{\kappa}(x)=\frac{i}{4}\left(\frac{\kappa}{2 \pi\|x\|}\right)^{d / 2-1} H_{d / 2-1}^{(1)}(\kappa\|x\|), \quad x \in \mathbb{R}^{d},
$$


where $H_{d / 2-1}^{(1)}$ is the Hankel function of the first kind [1]. In particular, in the case $d=3$, the corresponding fundamental solution is given by the classical Newton $(z=0)$, Yukawa $\left(-\Delta+z^{2}, z>0\right)$, or Helmholtz $\left(\Delta+\kappa^{2}, \kappa>0\right)$ kernels

$$
G_{0}(x):=\frac{1}{4 \pi} \frac{1}{\|x\|}, \quad G_{z}(x):=\frac{1}{4 \pi} \frac{e^{-z\|x\|}}{\|x\|}, \quad G_{\kappa}(x):=\frac{1}{4 \pi} \frac{e^{i \kappa\|x\|}}{\|x\|}, \quad x \in \mathbb{R}^{3},
$$

respectively.

The common feature of the family $G_{z}(\|x\|)$ is the analyticity in variable $\rho=\|x\|^{2}$, except for the singularity point $\rho=0$. This property together with spherical enables enables efficient separation of variables $x_{1}, \ldots, x_{d}$, in $\mathbb{R}^{d}$. In the case of nonoscillating kernels as in (2.15) the separable representation can be constructed by using the quadrature approximation of the Laplace transform

$$
G_{z}(\|x\|)=\int_{\mathbb{R}_{+}} \widehat{G}_{z}(t) e^{-t\|x\|^{2}} d t, \quad x \in \mathbb{R}^{d} .
$$

For $d=3$, the quadrature approximation of the Newton/Yukawa potentials $e^{-\kappa \sqrt{\rho}} / \sqrt{\rho}$ for $\kappa \in[0, \infty)$ is based on application of the sinc-quadratures to the Gauss transform

$$
G(\rho)=\frac{e^{-\kappa \sqrt{\rho}}}{\sqrt{\rho}}=\frac{2}{\sqrt{\pi}} \int_{\mathbb{R}_{+}} \exp \left(-\rho \tau^{2}-\kappa^{2} / 4 \tau^{2}\right) d \tau, \quad \rho=\|x\|^{2} .
$$

We are interested in the $L^{2}$-projection onto the set of tensor-product basis functions $\left\{\phi_{\mathbf{i}}\right\}$ introduced above (say, piecewise constant basis functions). The low tensor-rank approximations for the arising $d$ th order coefficients tensor

$$
\mathcal{G}=\left[G_{\mathbf{i}}\right]_{\mathbf{i} \in \mathcal{I}} \quad \text { with } G_{\mathbf{i}}=\int G(\rho(x)) \phi_{\mathbf{i}}(x) d x
$$

is proven in [12, Lemma 4.3], [19, Theorem 3]:

Proposition 2.3 For the tensor $\mathcal{G} \in \mathbb{R}^{\mathcal{I}}$ there exists the rank-R sinc-quadraturebased approximation $\mathcal{G}_{R} \in \mathcal{C}_{R, \mathbf{n}}$ such that

$$
\left\|\mathcal{G}-\mathcal{G}_{R}\right\| \leq C e^{-\beta R / \log R},
$$

where constants $C, \beta$ do not depend on $R$. The approximation error $\varepsilon>0$ is achieved by the Tucker/canonical approximation with the rank estimate $r \leq R \leq O(|\log \varepsilon|)$ up to low-order terms.

The numerical illustrations for $G_{z}(x)$ with $d=3$ can be found in Sect. 3.3 (see also $[2,19])$. Low rank representation of $G_{z}(x)$ can be utilized in fast tensor convolution transform [20, 21, 23]. Construction of separable approximation to the nonoscillating fundamental solution $G_{z}$ for $d>3$ can be based on similar sinc-methods.

In the present paper, we focus on the important special case. Specifically, we consider separable approximation for the Helmholtz kernel $G_{K}(x)$ for $d=3$ and for its formal multidimensional counterpart given by the function $e^{i \kappa\|x\|} /\|x\|, \quad x \in \mathbb{R}^{d}$, 
$d>3$ (though the latter does not represent the fundamental solution for the Helmholtz operator in $\mathbb{R}^{d}$, our constructions can be useful for understanding the effects of higher dimensions). Our main result proves the rank- $O(\kappa)$ tensor approximation for the Helmholtz kernel that scales linearly in the frequency parameter $\kappa$ (see Sect. 3.3). This result leads to sublinear cost $O(\kappa n \log n)$ with regard to $n \times n \times n$ spatial grids. To the best of our knowledge, low separation rank tensor approximation for oscillating kernels has not yet been addressed in the literature (cf. the asymptotically optimal methods in the volume size based on the fast multipole type representations [5, 6]).

In the next two subsections we discuss the particular numerical schemes for solving elliptic PDEs which can be gainfully improved by using tensor approximations to Green's functions.

\subsubsection{Application in FEM-BEM Coupling}

Consider the FEM applications dealing with elliptic problems in an unbounded domain. The initial BVP posed in $\Omega=\mathbb{R}^{d}$,

$$
-\Delta u+V u=f
$$

and subject to the corresponding radiation conditions as $\|x\| \rightarrow \infty$, can be reduced to the equation in the auxiliary bounded "tensor-product" domain $\Omega_{0}=[-a, a]^{d}, a>$ 0 , with the nonlocal boundary conditions posed on $\Gamma=\partial \Omega_{0}$. Specifically, assuming that $V(x)=$ const $\geq 0$ and $f(x)=0$, for $x \in \mathbb{R}^{d} \backslash \Omega_{0}$, and introducing the free-space Green's kernel $G(\|x-y\|)$ for the corresponding "external" elliptic operator with constant coefficients, (2.18) can be rewritten in the form

$$
-\Delta u+V u=f \quad \text { in } \Omega_{0} ; \quad u-(I+\mathcal{K})^{-1} \mathcal{V} \partial_{n} u=0 \quad \text { on } \Gamma,
$$

where the boundary Poincaré-Steklov (pseudo-differential) operator

$$
\mathcal{S}=(I+\mathcal{K})^{-1} \mathcal{V}: H^{-1 / 2}(\Gamma) \rightarrow H^{1 / 2}(\Gamma)
$$

is defined via the double layer and single layer integral operators $\mathcal{K}$ and $\mathcal{V}$ (see [25] for more details):

$$
\mathcal{K} u=\int_{\Gamma} \partial_{n, y} G(\|x-y\|) u(y) d y, \quad \mathcal{V} u=\int_{\Gamma} G(\|x-y\|) u(y) d y,
$$

with $\partial_{n}$ being the outward normal derivative on $\Gamma$. Hence the tensor-product representation of the integral operators $\mathcal{K}$ and $\mathcal{V}$ allows us to implement the nonlocal boundary conditions (and the whole solution process) in the combined equation (2.19) using tensor-product formats. In fact, if the Green function $G(\|x\|)$ allows a tensor-structured representation on a tensor grid in $\mathbb{R}^{d}$ that geometrically fits the boundary $\Gamma$, then the action of the corresponding boundary integral operators in (2.20) can be implemented in tensor format as well, just by "tracing" the tensorproduct volume representation onto the piecewise tensor-product boundary $\Gamma$. We will develop such tensor representations of the boundary (integral) operators elsewhere. In this way, the Poisson, Yukawa and Helmholtz type kernels in $\mathbb{R}^{d}$ can be adapted. 

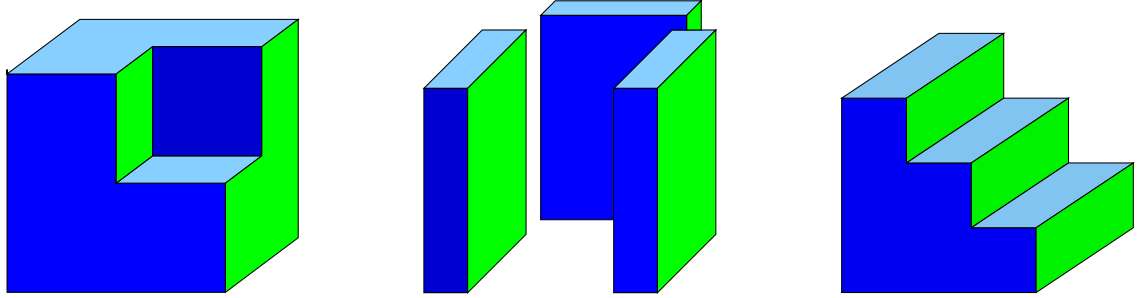

Fig. 1 Examples of step-type 3D geometries

The above strategy can also be extended to more complicated step-type geometries of $\Omega_{0}$. Figure 1 represents examples of 3D piecewise tensor-product geometries, which are well suited for tensor methods.

\subsubsection{The Green Function Formulation}

The Green function (fixed point) formulation applied to the spectral problems in quantum chemistry (the Lippmann-Schwinger equation) leads to the efficient numerical methods based on data-sparse representation of operators involved (see [4, 16] and references therein). This concept can also be applied as a preconditioning scheme for solving the elliptic boundary value problems.

The Green function formulations of the corresponding BVP and EVPs as in (2.14) can be derived by multiplication with the elliptic resolvent (Green's function operator) $R_{z}$. The corresponding integral (Green function) formulation of the elliptic BVP in the form $-\Delta u+V u=f$ reads as

$$
u=\mathcal{G}_{z} u+f \star G_{z}
$$

with

$$
\mathcal{G}_{z} u:=\left[V_{0} u\right] \star G_{z} \equiv R_{z}\left(V_{0} u\right), \quad V_{0}=z^{2}-V, R_{z}=\left(-\Delta+z^{2}\right)^{-1},
$$

where $\star$ denotes the convolution product in $L^{2}\left(\mathbb{R}^{d}\right)$. Here the parameter $z^{2} \geq 0$ can be specified via some optimization criteria; however, $z=0$ will be the standard choice (convolution with the Poisson kernel).

Under certain assumptions, $\mathcal{G}_{z}$ can be proven to be a bounded operator in $L^{2}\left(\mathbb{R}^{d}\right)$ (see $[4,27])$ that permits simple and robust iterative solution methods provided that the convolution transform can be computed in an efficient way (cf. [18]).

In the case of a spectral problem with the negative target eigenvalue $\lambda<0$, by setting $z=\sqrt{-\lambda}$ one obtains the integral formulation to the initial eigenvalue problem

$$
u=\mathcal{G}_{z} u \quad \text { with } \mathcal{G}_{z} u=\left[V_{0} u\right] * G_{z}
$$

The important feature of this formulation is that any eigenvalue-eigenfunction pair, $(\lambda, u),(\lambda$ in the discrete spectrum) for the operator $-\Delta+V$ generates a fixed point solution of the problem (2.22) (cf. [4, 16, 19] for the case of Hartree-Fock, electronic Schrödinger, and Kohn-Sham equations). 
We point out that the integral representation $\mathcal{G}_{z}=\left(V_{0} \cdot\right) \star G_{z}$ is computationally attractive due to the following advantageous features (see $[16,27])$ :

- Simple collocation-type discretization with discontinuous basis functions ( $L^{2}$ setting);

- Existence of low-separation rank approximations to the operators $V$ and $G_{z}$ that allow fast tensor-product convolution transform;

- Robust and fast convergence of the nonlinear fixed point iterations to solve (2.21) and (2.22), which are well suited for the numerical multilinear algebra via truncation to the prescribed tensor format.

For the rest of this section, we discuss in more detail the tensor approximation to the discrete convolution transform with the corresponding Green's kernels. Notice that the tensor-product multidimensional convolution for the class of translation invariant kernels was developed in [19, 20, 22]. In this way, we consider the collocationtype approximation of the operator $\mathcal{G}_{z}=\left(V_{0} \cdot\right) \star G_{z}$ with respect to the certain ansatz space $W=\operatorname{span}\left\{\phi_{\mathbf{i}}\right\} \subset L^{2}\left(\mathbb{R}^{d}\right)$ of possibly discontinuous tensor-product basis functions as in (2.10). Letting

$$
\psi=\sum_{\mathbf{i} \in \mathcal{I}} a_{\mathbf{i}} \phi_{\mathbf{i}}, \quad \mathbb{V}_{0}=\left\{\left\langle V_{0} \phi_{\mathbf{i}}, \phi_{\mathbf{j}}\right\rangle\right\}_{\mathbf{i}, \mathbf{j} \in \mathcal{I}} \in \mathbb{R}^{\mathcal{I} \times \mathcal{I}},
$$

we calculate the $L^{2}$-projection $\mathcal{P}_{W}\left(V_{0} \psi\right)$ of $V_{0} \psi$ onto $W$,

$$
\mathcal{P}_{W}\left(V_{0} \psi\right)=\sum_{\mathbf{j} \in \mathcal{I}} \sum_{\mathbf{i} \in \mathcal{I}} a_{\mathbf{i}}\left\langle V_{0} \phi_{\mathbf{i}}, \phi_{\mathbf{j}}\right\rangle \phi_{\mathbf{j}}=\sum_{\mathbf{j} \in \mathcal{I}} b_{\mathbf{j}} \phi_{\mathbf{j}}
$$

with $b_{\mathbf{j}}=\left(\mathbb{V}_{0} \mathcal{A}\right)_{\mathbf{j}}, \mathcal{A}=\left[a_{\mathbf{i}}\right]$. Introducing the coefficients tensor

$$
\mathcal{B}=\left[b_{\mathbf{j}}\right]_{\mathbf{j} \in \mathcal{I}} \in \mathbb{R}^{\mathcal{I}}, \quad \text { i.e., } \quad \mathcal{B}=\mathbb{V}_{0} \mathcal{A},
$$

the discretization of $\mathcal{G}_{z} \psi$ can then be defined by multidimensional convolution

$$
\mathcal{G}_{z} \psi \approx \mathcal{G} \star \mathcal{B}
$$

where tensor $\mathcal{G}$ is obtained by the projection of the convolving kernel $G_{z}(x)=$ $e^{-z\|x\|} /\|x\|$ onto the space $W$ (see Proposition 2.3):

$$
\mathcal{G}=\left[G_{\mathbf{i}}\right]_{\mathbf{i} \in \mathcal{I}} \quad \text { with } G_{\mathbf{i}}=\int G_{z}(x) \phi_{\mathbf{i}}(x) d x .
$$

In the case of piecewise constant basis functions in (2.10) the stiffness matrix $\mathbb{V}_{0}$ becomes diagonal, $\mathbb{V}_{0}=\operatorname{diag}\left\{\left\langle V_{0} \phi_{\mathbf{i}}, \phi_{\mathbf{i}}\right\rangle\right\}_{\mathbf{i} \in \mathcal{I}}$ (local operator of multiplication with $V_{0}(x)$ ). Here we presuppose the existence of low Kronecker rank representation for the matrix $\mathbb{V}_{0}$ as in (2.6). For example, this is the case if $V_{0}(x)$ is given by $\sum_{v} \frac{Z_{v}}{\left\|x-x_{v}\right\|}+g(x)$, with a smooth function $g$.

In some physical models, solution (2.14) exhibits exponential decay as $\|x\| \rightarrow \infty$. In this case, the computational domain can be restricted to the fixed-size hypercube in $\mathbb{R}^{d}$. A similar situation arises in the traditional 3D BEM applications (see Sect. 2.4.2 above). 


\section{Low Tensor-Rank Preconditioners}

\subsection{Analysis of the Preconditioner $\mathcal{B}_{R}$}

We utilize the rank- $R$ preconditioner $\mathcal{B}_{R}$ based on the sinc-quadrature approximation as follows:

$$
\mathcal{B}_{R}:=\sum_{k=-M}^{M} c_{k} \bigotimes_{\ell=1}^{d} \exp \left(-t_{k} \mathcal{A}_{\ell}\right) \approx \mathcal{L}_{0}^{-1} \quad\left(t_{k}, c_{k} \in \mathbb{R}_{+}, \mathcal{A}_{\ell} \in \mathbb{R}^{n \times n}\right) .
$$

It can be proven to provide exponential convergence in $R=2 M+1$ (see Lemma 3.2 below). The next simple lemma proves the linear scaling of the preconditioner $\mathcal{B}_{R}$ in $d$.

Lemma 3.1 (Linear Scaling) Preconditioner $\mathcal{B}_{R}$ in (3.1) has the Kronecker rank $R=2 M+1$, i.e., we have

$$
\mathcal{B}_{R} \in \mathcal{M}_{R, \mathbf{n}} \text {. }
$$

Let the operator coefficients $D_{\ell}, V_{\ell}(\ell=1, \ldots, d)$ be constant. Then the required storage and respective cost of the matrix-vector multiplication with rank-1 tensor can be estimated by

$$
\mathcal{O}(d R n) \quad \text { and } \mathcal{O}(d R n \log n) .
$$

In the case of variable coefficients the related cost is bounded, respectively, by

$$
\mathcal{O}\left(d R n^{2}\right) \text { and } \mathcal{O}\left(d R n^{2}\right) .
$$

Proof The Kronecker rank estimate is due to representation (3.1). Taking into account that in the case of constant coefficients the Galerkin matrix $\mathcal{A}_{\ell}(\ell=1, \ldots, d)$ can be diagonalized at the $\operatorname{cost} \mathcal{O}(n \log n)$ by using FFT matrices, we arrive at the linear-logarithmic scaling in $n$ for the related matrix operations. The last assertion is due to the full format representation of the matrix exponential $\exp \left(-t_{k} \mathcal{A}_{\ell}\right) \in \mathbb{R}^{n \times n}$ in the general case.

To control the quality of preconditioning, we make use of the known error bound for the sinc-quadrature (3.1) applied to the Laplace transform of the matrix-valued function

$$
\mathcal{L}_{0}^{-1}=\int_{0}^{\infty} e^{-t \mathcal{L}_{0}} d t \quad \text { with } \operatorname{cond}\left(\mathcal{L}_{0}\right)=O\left(n^{2}\right) .
$$

The appropriate choice of quadrature parameters $t_{k}, c_{k}$ (see (3.3)) leads to the exponential convergence rate (cf. [12, Lemma 4.3])

$$
\left\|\mathcal{L}_{0}^{-1}-\mathcal{B}_{R}\right\| \leq C_{0} e^{-\pi \sqrt{M}}
$$

where the constant $C_{0}>0$ does not depend on $M$ or on $n$. Notice that a similar result was proven in [4, Theorem 22]. The following lemma proves the spectral equivalence estimates: 
Lemma 3.2 (Spectral Equivalence) Let us set in (3.1)

$$
t_{k}=e^{k \mathfrak{h}}, \quad c_{k}=\mathfrak{h} t_{k}, \quad \mathfrak{h}=\pi / \sqrt{M}, \quad k=-M, \ldots M,
$$

and suppose that the constants $C_{0}, C_{1}, C_{2}$ are determined by (3.2) and (2.13), respectively. Choose $M$ such that the inequality $C_{0}\left\|\mathcal{L}_{0}\right\|\left\|\mathcal{B}_{R}^{-1}\right\| e^{-\pi \sqrt{M}}<q(M) C_{1}$ holds with $q<1$. Then we have

$$
\widetilde{C}_{1}\langle\mathcal{L} U, U\rangle \leq\left\langle\mathcal{B}_{R}^{-1} U, U\right\rangle \leq \widetilde{C}_{2}\langle\mathcal{L} U, U\rangle, \quad \forall U \in \mathbb{V}_{n},
$$

with spectral equivalence constants $\widetilde{C}_{1}, \widetilde{C}_{2}>0$ that allow the following bound on the condition number:

$$
\frac{\widetilde{C}_{2}}{\widetilde{C}_{1}} \leq \frac{1}{1-q(M)} \frac{C_{2}}{C_{1}}+\frac{q(M)}{1-q(M)} .
$$

Proof The matrices $\mathcal{A}_{\ell}$ are symmetric and positive definite; hence the Laplace transform and related exponential factors in (3.1) are correctly defined. Moreover, all terms in the sum $\mathcal{L}_{0}=\mathcal{A}_{1} \otimes \cdots \otimes I+\cdots+I \otimes \cdots \otimes \mathcal{A}_{d}$ mutually commute providing the corresponding factorization of the matrix exponents $e^{-t_{k} \mathcal{L}_{0}}=$ $\prod_{\ell=1}^{d} e^{-t_{k} I \otimes \cdots \otimes \mathcal{A}_{\ell} \otimes \cdots \otimes I}(k=-M, \ldots, M)$. Then using the property of matrix exponential we obtain

$$
\prod_{\ell=1}^{d} e^{-t_{k} I \otimes \cdots \otimes \mathcal{A}_{\ell} \otimes \cdots \otimes I}=\bigotimes_{\ell=1}^{d} e^{-t_{k} \mathcal{A}_{\ell}},
$$

see [17, Theorem 5.3]. Moreover, we have

$$
\left\|\mathcal{L}_{0}-\mathcal{B}_{R}^{-1}\right\|=\left\|-\mathcal{L}_{0}\left(\mathcal{L}_{0}^{-1}-\mathcal{B}_{R}\right) \mathcal{B}_{R}^{-1}\right\| \leq\left\|\mathcal{L}_{0}\right\|\left\|\mathcal{L}_{0}^{-1}-\mathcal{B}_{R}\right\|\left\|\mathcal{B}_{R}^{-1}\right\|
$$

Using (3.2) the constants $\widetilde{C}_{1}, \widetilde{C}_{2}>0$ can be estimated by

$$
C_{1}-C_{0}\left\|\mathcal{L}_{0}\right\|\left\|\mathcal{B}_{R}^{-1}\right\| e^{-\pi \sqrt{M}} \leq \widetilde{C}_{1}, \quad \widetilde{C}_{2} \leq C_{2}+C_{0}\left\|\mathcal{L}_{0}\right\|\left\|\mathcal{B}_{R}^{-1}\right\| e^{-\pi \sqrt{M}},
$$

with $C_{0}>0$ defined in (3.2). Combining the above inequality with the error estimate (3.2) and with (2.13) leads to the desired bound.

Remark 3.3 Lemma 3.2 indicates that the Kronecker rank- $R$ preconditioner $\mathcal{B}_{R}^{-1}$ has linear (or quadratic) scaling in the univariate problem size $n$, providing at the same time the condition number of order $C_{2} / C_{1}$ in (2.13), as soon as the estimate

$$
C_{0}\left\|\mathcal{L}_{0}\right\|\left\|\mathcal{B}_{R}^{-1}\right\| e^{-\pi \sqrt{M}}<C_{1}
$$

holds. The latter is valid for $R=O\left(\left|\log \left(q(M) / C_{1}\right)\right|^{2}\right)=O\left((\log n)^{2}\right)$. Notice that the modified sinc-quadrature leads to the improved convergence rate in (3.2), $C e^{-\alpha M / \log (M)}$ with $\alpha=\log \left(\operatorname{cond}\left(\mathcal{L}_{0}\right)\right)$ (cf. [17, Lemma 9.3], [12, Lemma 4.3]), again providing the rank estimate $R=O\left(\log \left(|\log (q(M))| / C_{1}\right)\right)=O\left((\log h)^{2}\right)$. 

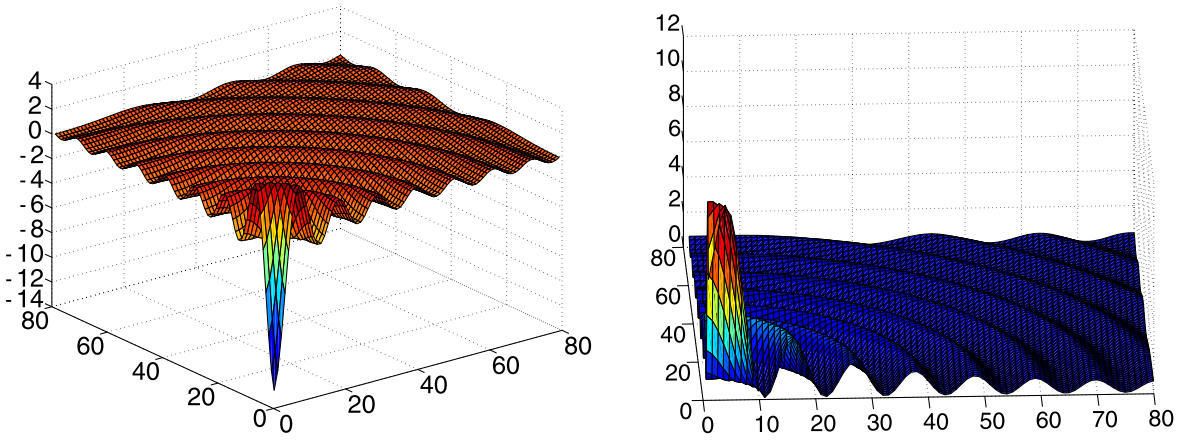

Fig. 2 Visualization of $f_{1, \kappa}, f_{2, \kappa}$ for $\kappa=15, d=3$

\subsection{Canonical Approximation of the Oscillating Helmholtz Kernels}

We propose the construction of exponentially convergent tensor decompositions of the weakly singular oscillating kernels $e^{i \kappa\|x-y\|} /\|x-y\|, \kappa \in \mathbb{R}$, such that its real and imaginary parts

$$
\frac{\cos (\kappa\|x-y\|)}{\|x-y\|} \text { and } \frac{\sin (\kappa\|x-y\|)}{\|x-y\|}, \quad x, y \in \mathbb{R}^{d},
$$

will be treated separately. In the case $d=3$, we obtain the rank- $O(\kappa)$ separable approximation for the oscillating Helmholtz kernel. At the end of Sect. 3 we provide numerical illustrations for $d=3$, and for moderate $\kappa \leq 15$ which may substantiate the generic constants in the theoretical rank estimates.

On the first step, we transform the singular cos-kernel to the "more regular" kernel by subtraction of the principal singularity $1 /\|x\|$. Then we construct separable approximations of the "regular" Helmholtz potentials

$$
f_{1, \kappa}(\|x\|):=\frac{\sin (\kappa\|x\|)}{\|x\|} ; \quad f_{2, \kappa}(\|x\|):=\frac{1}{\|x\|}-\frac{\cos (\kappa\|x\|)}{\|x\|}=\frac{2 \sin ^{2}\left(\frac{\kappa}{2}\|x\|\right)}{\|x\|},
$$

which leads to the respective approximations of the related kernel functions

$$
f_{1, \kappa}(\|x-y\|), \quad f_{2, \kappa}(\|x-y\|), \quad x, y \in \mathbb{R}^{d} .
$$

Figure 2 shows the complicated shape of the target functions $f_{1, \kappa}$ and $f_{2, \kappa}$ (2D slices of the potential in $d=3$ ).

In the next statements we prove the rank estimates for the tensor approximations of both $f_{1, \kappa}$ and $f_{2, \kappa}$.

Theorem 3.4 For given tolerance $\varepsilon>0$, the function $f_{1, \kappa}:\left[0, \frac{2 \pi}{\sqrt{d}}\right]^{d} \rightarrow \mathbb{R}$ admits the Tucker/canonical approximations such that

$$
\sigma\left(f_{1, \kappa}, \mathcal{S}\right) \leq C \varepsilon \quad \text { with } \mathcal{S}=\left\{\mathcal{T}_{\mathbf{r}}, \mathcal{C}_{R}\right\}
$$


and with the rank estimates $(\mathbf{r}=(r, \ldots, r))$,

$$
r \leq R \leq C d(|\log \varepsilon|+\kappa) .
$$

Proof We set $t=\|x\|^{2}$ and then approximate the entire function $g(t)=\frac{\sin (\kappa \sqrt{t})}{\sqrt{t}}$, $t \in[0,2 \pi]$ by trigonometric polynomials in $t$ up to the accuracy $\varepsilon$ in the max-norm. To that end we make use of the change of variables $z=\cos (t), z \in[-1,1]$, and consider the entire function $f(z)=g(\arccos (z))$ that has the maximum value $O\left(e^{\kappa}\right)$ on the respective Bernstein's regularity ellipse of size $O(1)$. Applying the Chebyshev series to the function $f(z)$,

$$
f(z) \approx C_{0}+\sum_{m=1}^{M} C_{m} T_{m}(z), \quad z \in[-1,1],
$$

and taking into account that the Chebyshev polynomials satisfy $T_{m}(z)=$ $\cos (m \operatorname{arrcos} z), z \in[-1,1]$, we are led to approximation by trigonometric polynomials (up to tolerance $\varepsilon>0$ ) with $M=O(|\log \varepsilon|+\kappa)$ terms, where each trigonometric term has the form $\cos (m t)=\cos \left(m\|x\|^{2}\right)$.

Notice that the multivariate function $h(x):=\cos \left(x_{1}^{2}+\cdots+x_{d}^{2}\right)$ has a separation rank $R \leq d$, i.e., $h \in \mathcal{C}_{R}$, that is the consequence of the "magic" rank- $d$ representation (cf. [3])

$$
\sin \left(\sum_{i=1}^{d} x_{j}\right)=\sum_{i=1}^{d} \sin x_{j} \prod_{k \in\{1, \ldots, d\} \backslash\{j\}} \frac{\sin \left(x_{k}+\alpha_{k}-\alpha_{j}\right)}{\sin \left(\alpha_{k}-\alpha_{j}\right)},
$$

which is valid for all $\alpha_{k} \in \mathbb{R}$, such that $\sin \left(\alpha_{k}-\alpha_{j}\right) \neq 0$ for all $j \neq k$.

Now the result follows by combination of the rank estimate for Chebyshev trigonometric approximation with the inclusion $h \in \mathcal{C}_{R}$.

Theorem 3.4 gives the constructive proof of the existence of low tensor-rank approximation for the continuous function $f_{1, \kappa}$. The next statement applies to the $d$ th order tensor representing the projected kernel $f_{2, \kappa}$ onto the piecewise constant basis functions. Extension to the case of higher-order tensor-product polynomials is straightforward. For further constructions, we introduce the function

$$
f_{1}(t):=\frac{\sin ^{2}(\kappa / 2 \sqrt{t})}{t} \equiv\left(f_{1, \kappa / 2}(t)\right)^{2} .
$$

Theorem 3.5 For given $\varepsilon>0$, the coefficients tensor corresponding to $f_{2, \kappa}$ : $\left[0, \frac{2 \pi}{\sqrt{d}}\right]^{d} \rightarrow \mathbb{R}$

$$
\mathcal{G}=\left[G_{\mathbf{i}}\right]_{\mathbf{i} \in \mathcal{I}} \quad \text { with } G_{\mathbf{i}}=\left\|x_{\mathbf{i}}\right\| f_{1}\left(\left\|x_{\mathbf{i}}\right\|\right) \int_{\mathbb{R}^{d}} \frac{1}{\|x\|} \phi_{\mathbf{i}}(x) d x
$$

admit Tucker/canonical approximations such that $\sigma(\mathcal{G}, \mathcal{S}) \leq C \varepsilon$ with $\mathcal{S}=\left\{\mathcal{T}_{\mathbf{r}}, \mathcal{C}_{R}\right\}$ and with the rank estimates

$$
r \leq R \leq C d^{2}|\log \varepsilon|(|\log \varepsilon|+\kappa) \quad(\mathbf{r}=(r, \ldots, r)) .
$$


The numerical complexity of the rank- $R$ canonical approximation is bounded by

$$
O\left(d^{3}|\log \varepsilon|(|\log \varepsilon|+\kappa) n\right) .
$$

Proof We write the function $g(t)=\frac{\sin ^{2}(\kappa / 2 \sqrt{t})}{\sqrt{t}}$ (with $t=\|x\|^{2} \in[0,2 \pi]$ ) as

$$
g(t)=t \frac{1}{\sqrt{t}} f_{1}(t) \quad \text { with } f_{1}(t)=\left(f_{1, \kappa / 2}(t)\right)^{2} .
$$

Now we apply the same argument as in Theorem 3.4 to the entire function $f_{1}$ : $[0,2 \pi] \rightarrow \mathbb{R}$ and obtain its separable approximation in classes $\mathcal{T}_{\mathbf{r}}$ and $\mathcal{C}_{R}$ (on the continuous level) that admits the $\kappa$-dependent rank estimate

$$
r \leq R \leq C d(|\log \varepsilon|+\kappa) .
$$

Since $f=t$ is the rank- $d$ function of variables $\left(x_{1}, \ldots, x_{d}\right)$, we are left to the tensor approximation of the coefficients tensor for the Newton potential 1/\|x\| (see Proposition 2.3, [18, Lemma 4.3] and [19, Theorem 3]). Using the rank- $|\log \varepsilon|$ approximation of the coefficients tensor for Newton potential, we arrive at the desired rank estimate for the resultant canonical tensor $\left[G_{\mathbf{i}}\right]_{\mathbf{i} \in \mathcal{I}}$ obtained as the Hadamard product of three canonical factors with respective rank parameters $d,|\log \varepsilon|$, and $d(|\log \varepsilon|+\kappa)$.

We comment that Theorems 3.4 and 3.5 indicate linear scaling of the tensor rank in the frequency parameter $\kappa$, which can lead to the remarkable reduction of the numerical cost in the case of moderate and high frequencies, $\kappa \leq C n$.

\subsection{Complexity Issues and Numerics}

Theorems 3.4 and 3.5 imply that the canonical tensor representation (up to tolerance $\varepsilon>0$ ) of the discretized Helmholtz kernel on an $n \times n \times n$ grid amounts to $O(|\log \varepsilon|(|\log \varepsilon|+\kappa) n)$ which allows the upper bound $O\left(|\log \varepsilon| n^{2}\right)$ for high frequency regime, $\kappa=C n$.

Given the dimension parameter $d$ and the univariate grid size $n$, we ask the question: When does the canonical format require less storage than the orthogonal Tucker representation. The corresponding parameter relation is given by

$$
d R n \leq r^{d}+d r n,
$$

which leads to the condition that ensures the priority of the canonical representation

$$
d\left(\frac{R}{r}-1\right) n \leq r^{d-1} .
$$

The latter will always be satisfied in higher dimensions.

In the case of traditional FEM/BEM applications, i.e., if $d=3$, we set $\frac{R}{r}=2$ (typical in our numerical practice), and then we arrive at the priority relation for the canonical representation

$$
\sqrt{3 n} \leq r
$$



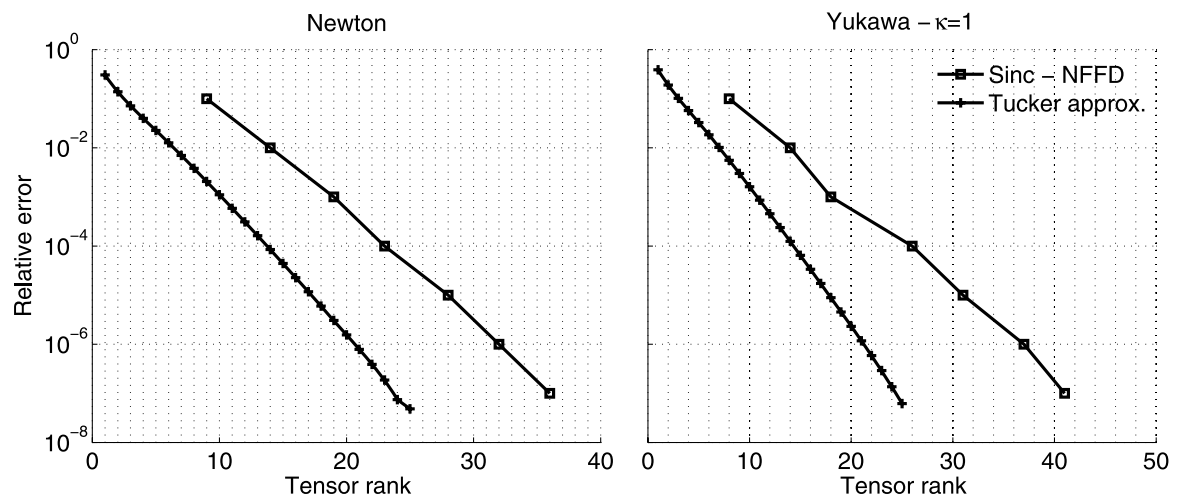

Fig. 3 The Tucker vs. canonical approximation of the 3D Newton/Yukawa kernels
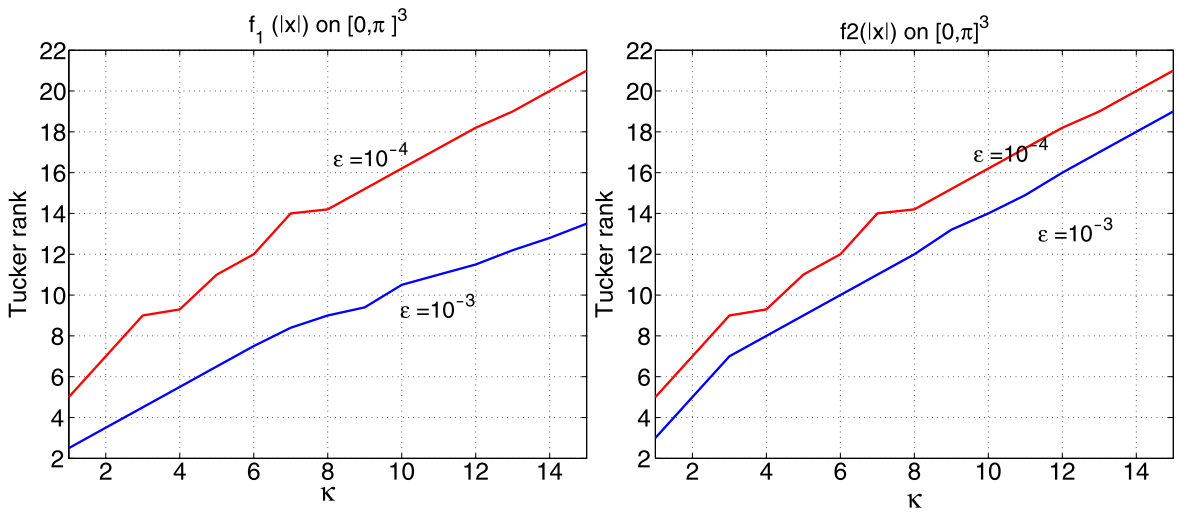

Fig. 4 Convergence history for the Tucker model applied to $f_{1, \kappa}, f_{2, \kappa}, \kappa \in[1,15]$

which can be satisfied only on very coarse grids (recall that for nonoscillating kernels we have $r=O(\log n))$. However, for the case of oscillating kernels we may have $\kappa=C n$, i.e., the canonical decomposition would be preferable.

Proceeding with the case of the 3D Helmholtz kernel, we notice that $\kappa \leq C n$ ensures that the Tucker model scales linear in $N_{v o l}=n^{3}$. Furthermore, if $\kappa \leq C n^{2 / 3}$, then the Tucker model scales sublinear in $N_{v o l}$ and linear in $N_{B E M}=n^{2}$. Recall that the approximation condition in the high-frequency domain is given by $\kappa \leq C n$.

Below we present some numerical illustrations. The computations were performed in MATLAB 7.3.

Example 1 Figure 3 represents the convergence history for the best orthogonal Tucker [23] vs. canonical [2] approximations of the Newton/Yukawa potentials on an $n \times n \times n$ grid for $n=2048$.

Example 2 Figure 4 shows the convergence history for the Tucker model applied to $f_{1, \kappa}, f_{2, \kappa}$ depending on $\kappa \in[1,15]$ and termination criteria with fixed values $\varepsilon_{1}>0$ 

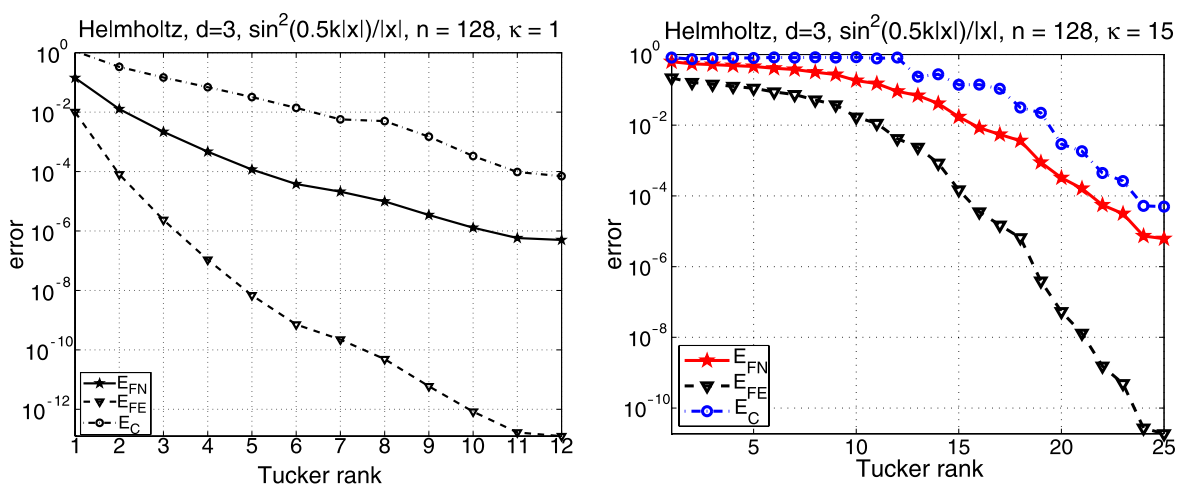

Fig. 5 Convergence history for the Tucker model applied to $f_{2, \kappa}, \kappa=1,15$
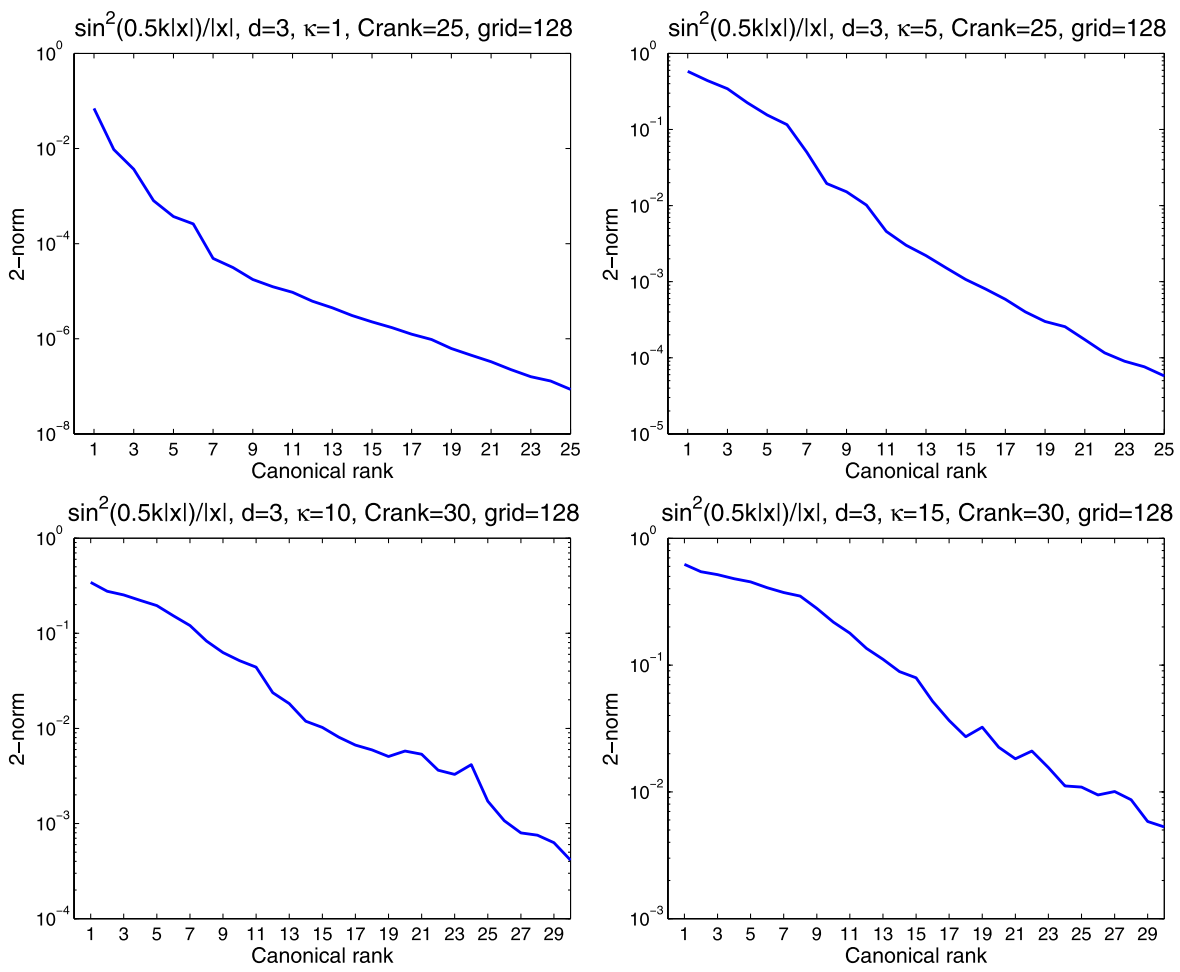

Fig. 6 Convergence history for the canonical model applied to $f_{2, \kappa}, \kappa \in[1,15]$

and $\varepsilon_{2}>0$. It is clearly indicating the relation $r \sim C+\kappa$ for different (fixed) values of $\varepsilon_{1}=10^{-3}$ and $\varepsilon_{2}=10^{-3}$.

Convergence for the Tucker and canonical models applied to $f_{2, \kappa}, \kappa \in[1,15]$ is presented in Figures 5 and 6, respectively. 

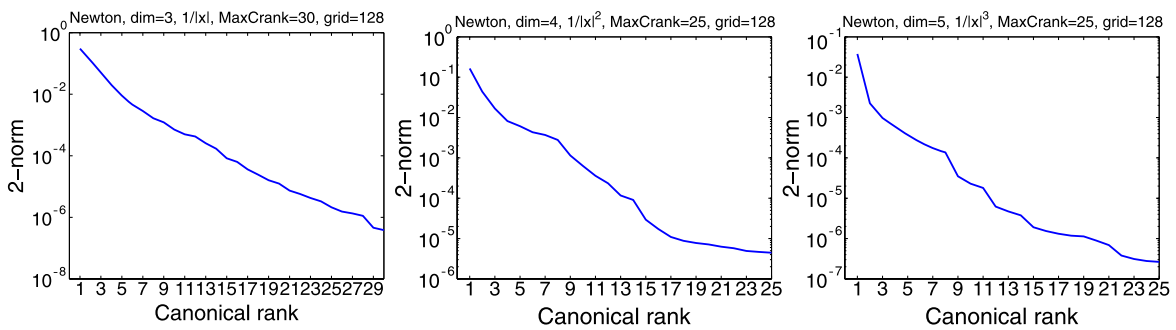

Fig. 7 Canonical approximation applied to the Newton potential $1 /\|x\|^{d-2}$ for $d=3,4,5$
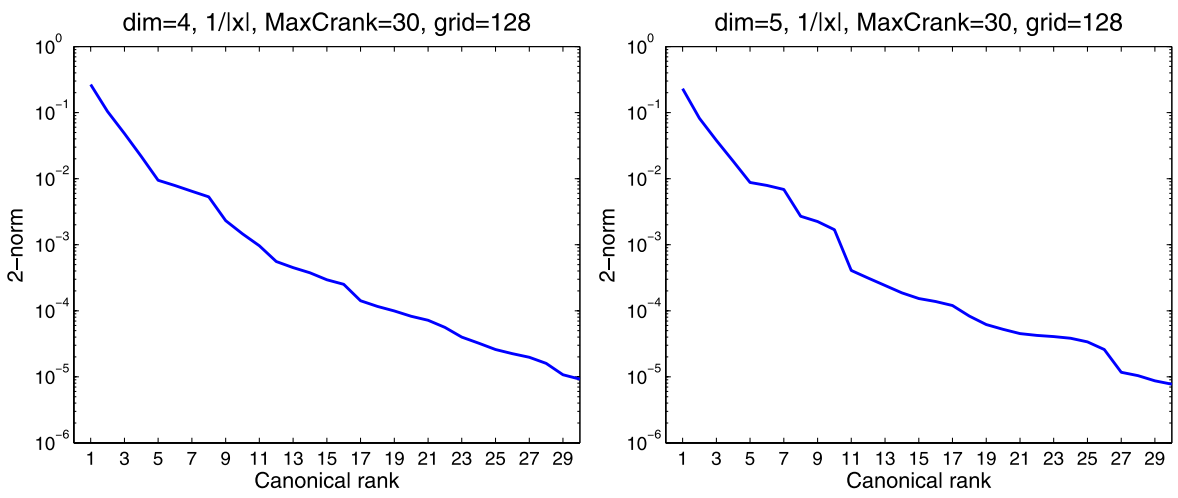

Fig. 8 Canonical approximation applied to the weakly singular potential $1 /\|x\|$ for $d=4,5$

Figure 5, right clearly demonstrates exponential convergence in the Tucker rank $r$ in the interval $r \geq r_{0}=\kappa$ (supports the theory). We obtain the canonical approximation (cf. Fig. 6) by using the two-level format $\mathcal{T}_{\mathcal{C}_{R, \mathrm{r}}}$, i.e., we compute the canonical rank- $R$ decomposition by ALS-type iteration applied to the small-size $r \times r \times r$ Tucker core.

Example 3 Results for canonical approximation of the Newton potential $1 /\|x\|^{d-2}$, $x \in \mathbb{R}^{d}$, for $d=3,4,5$, are depicted in Figure 7 , while the respective convergence rate for weakly singular potential $1 /\|x\|, d=4,5$, can be seen in Figure 8 .

Example 4 Next, Figure 9 indicates that the convergence of spectral (via trigonometric functions) approximations of the Helmholtz kernel deteriorates dramatically even for moderate frequencies.

Example 5 Finally, we present the results on the iterative calculation of the minimal eigenvalue for the $d$-dimensional finite difference Laplacian by power method with the rank truncation [13]. The rank- $R$ tensor approximation is given by (3.1) with $\mathcal{A}_{\ell}=\operatorname{tridiag}\{-1,2,-1\} \in \mathbb{R}^{n \times n}$. Here, we discretize the problem on $(0, \pi)^{d}$ using $n^{d}$ grid points and apply the sinc-quadrature with $M=49$ to obtain the rank$(2 M+1)$ approximation of the Laplacian inverse for $d=3,10,50$. The next table presents the computational time (in seconds) per iteration, the relative $H^{1}$-error in 

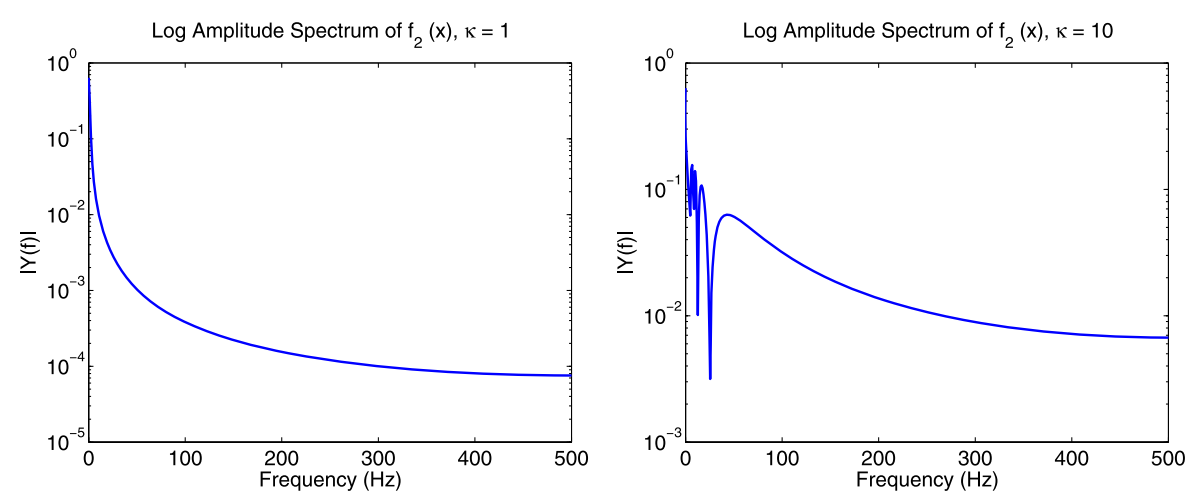

Fig. 9 On spectral approximation of the Helmholtz kernel

Table 1 Minimal eigenvalue for the $d$-dimensional Laplacian $(d=3,10,50)$

\begin{tabular}{rlll}
\hline$d$ & Time/it & $\delta_{\lambda}$ & $\delta_{u}$ \\
\hline 3 & 0.9 & $3.1 \times 10^{-6}$ & $4.5 \times 10^{-4}$ \\
10 & 2.9 & $3.1 \times 10^{-6}$ & $3.8 \times 10^{-4}$ \\
50 & 14.7 & $3.1 \times 10^{-6}$ & $3.1 \times 10^{-4}$
\end{tabular}

the eigenfunction, and the relative error in the eigenvalue for $n=2^{9}$. In all cases, the number of power iterations does not exceed 6 .

Table 1 clearly indicates the linear scaling in $d$ of tensor-product approximation. More detailed numerical illustrations on tensor-structured eigenvalue solvers can be found in [13].

Acknowledgements The author is thankful to Prof. S. Sauter (University of Zurich) and Prof. Ch. Schwab (ETH, Zurich) for helpful discussions on tensor methods in high dimensional FEM/BEM applications. This work was partially supported by FIM, ETH Zurich (May-July, 2008).

\section{References}

1. Abramowitz, M., Stegun, I.A.: Handbook of Mathematical Functions. Dover, New York (1968)

2. Bertoglio, C., Khoromskij, B.N.: Low rank tensor-product approximation of the projected Green kernels via Sinc-quadratures. Preprint MPI MIS 79/2008, Leipzig, 2008 (submitted)

3. Beylkin, G., Mohlenkamp, M.J.: Numerical operator calculus in higher dimensions. Proc. Natl. Acad. Sci. USA 99, 10246-10251 (2002)

4. Beylkin, G., Mohlenkamp, M.J., Pérez, F.: Approximating a wavefunction as an unconstrained sum of Slater determinants. J. Math. Phys. 49, 032107 (2008)

5. Beylkin, G., Kurcz, Ch., Monzón, L.: Fast algorithms for Helmholtz Green's function. Proc. R. Soc., Ser. A 464, 3301-3326 (2008)

6. Beylkin, G., Kurcz, Ch., Monzón, L.: Fast Convolution with the Free Space Helmholtz Green's function. University of Colorado at Bolder, http://amath.colorado.edu/pub/wavelets/papers/ BE-KU-MO-2008P.pdf

7. De Lathauwer, L., De Moor, B., Vandewalle, J.: On the best rank-1 and rank- $\left(R_{1}, \ldots, R_{N}\right)$ approximation of higher-order tensors. SIAM J. Matrix Anal. Appl. 21, 1324-1342 (2000) 
8. De Lathauwer, L., De Moor, B., Vandewalle, J.: A multilinear singular value decomposition. SIAM J. Matrix Anal. Appl. 21, 1253-1278 (2000)

9. Gavrilyuk, I.P., Hackbusch, W., Khoromskij, B.N.: $\mathcal{H}$-matrix approximation for the operator exponential with applications. Numer. Math. 92, 83-111 (2002)

10. Gavrilyuk, I.P., Hackbusch, W., Khoromskij, B.N.: Tensor-product approximation to elliptic and parabolic solution operators in higher dimensions. Computing 74, 131-157 (2005)

11. Griebel, M., Hamaekers, J.: Sparse grids for the Schrödinger equation. ESAIM: M2AN 41, 215-247 (2007)

12. Hackbusch, W., Khoromskij, B.N.: Low-rank Kronecker product approximation to multi-dimensional nonlocal operators, part I: separable approximation of multi-variate functions. Computing 76, 177202 (2006)

13. Hackbusch, W., Khoromskij, B.N., Sauter, S., Tyrtyshnikov, E.: Tensor formats in elliptic problems. Preprint MPI MIS 78/2008, Leipzig, 2008 (SINUM, submitted)

14. Hackbusch, W., Khoromskij, B.N., Tyrtyshnikov, E.: Hierarchical Kronecker tensor-product approximation. J. Numer. Math. 13, 119-156 (2005)

15. Hackbusch, W., Khoromskij, B.N., Tyrtyshnikov, E.E.: Approximate Iterations for Structured Matrices. Numer. Math. 109, 365-383 (2008)

16. Harrison, R.J., Fann, G.I., Yanai, T., Gan, Z., Beylkin, G.: Multiresolution quantum chemistry: basic theory and initial applications. J. Chem. Phys. 121(23), 11587-11598 (2004)

17. Khoromskij, B.N.: An Introduction to Structured Tensor-Product Representation of Discrete Nonlocal Operators, vol. 27. Max-Planck-Institut für Mathematik in den Naturwissenschaften, Leipzig (2005)

18. Khoromskij, B.N.: Structured rank- $\left(r_{1}, \ldots, r_{d}\right)$ decomposition of function-related tensors in $\mathbb{R}^{d}$. Comput. Methods Appl. Math. 6(2), 194-220 (2006)

19. Khoromskij, B.N.: On tensor approximation of green iterations for Kohn-Sham equations. Comput. Vis. Sci. 11, 259-271 (2008). doi:10.1007/s00791-008-0097-X

20. Khoromskij, B.N.: Fast and accurate tensor approximation of multivariate convolution with linear scaling in dimension. Preprint MPI MIS 36, Leipzig, 2008. J. Comput. Appl. Math. (accepted)

21. Khoromskij, B.N., Khoromskaia, V., Chinnamsetty, S.R., Flad, H.-J.: Tensor decomposition in electronic structure calculations on 3D Cartesian grids. J. Comput. Phys. 228, 5749-5762 (2009).

22. Khoromskij, B.N., Khoromskaia, V.: Low rank tucker-type tensor approximation to classical potentials. Cent. Eur. J. Math. 5(3), 1-28 (2007)

23. Khoromskij, B.N., Khoromskaia, V.: Multigrid accelerated tensor approximation of function related multi-dimensional arrays. SIAM J. Sci. Comput. 31(4), 3002-3028 (2009)

24. Khoromskij, B.N., Schwab, Ch.: Tensor approximation of multi-parametric elliptic problems in stochastic PDEs. ETH Zurich, 2009 (in preparation)

25. Khoromskij, B.N., Wittum, G.: Numerical Solution of Elliptic Differential Equations by Reduction to the Interface. LNCSE, vol. 36. Springer, Berlin (2004)

26. Maz'ya, V., Schmidt, G.: Approximate Approximations. Math. Surveys and Monographs, vol. 141. AMS, Providence (2007)

27. Mohlenkamp, M., Young, M.J.: Convergence of Green's iterations for Schrödinger equations. Preprint 2007 (to appear)

28. Reed, M., Simon, B.: Methods of Modern Mathematical Physics, I: Functional Analysis. Academic Press, New York (1972)

29. Stenger, F.: Numerical Methods Based on Sinc and Analytic Functions. Springer, Berlin (1993)

30. Todor, R.-A., Schwab, Ch.: Convergence rate for sparse approximation of elliptic problems with stochastic coefficients. IMA J. Numer. Anal. 27, 232-261 (2007) 